\title{
The Increased Frequency of Carbapenem Resistant Non Fermenting Gram Negative Pathogens as Causes of Health Care Associated Infections in Adult Cancer Patients
}

\author{
Hadir A. El-Mahallawy1, Rasha M. Abdel Hamid1, Safaa Shawky Hassan1*, Samah Radwan1, \\ Magdy Saber ${ }^{2}$ \\ ${ }^{1}$ Clinical Pathology Department, National Cancer Institute, Cairo University, Giza, Egypt \\ ${ }^{2}$ Medical Oncology Department, National Cancer Institute, Cairo University, Giza, Egypt \\ Email: "safaa_shawky@hotmil.com
}

Received 7 August 2015; accepted 18 September 2015; published 21 September 2015

Copyright (C) 2015 by authors and Scientific Research Publishing Inc.

This work is licensed under the Creative Commons Attribution International License (CC BY).

http://creativecommons.org/licenses/by/4.0/

(c) (i) Open Access

\section{Abstract}

Background and Aim: Multi drug resistant Non fermenting gram negative bacilli (NFGNB) have emerged as a major cause of health-care associated infections especially in immunocompromised hosts. The aim of the study was to investigate the prevalence of NFGNB as a cause of health-care associated infections (HAI) in cancer patients and determine their resistance pattern. Patients and Methods: During the study period, 158 NFGNB isolates were collected. Microscan Walk Away 9 was used for identification and testing for the metallo- $\beta$-lactamases (MBLs) was done by ImipenemEDTA combined disk synergy test (CDST-IPM). Results: NFGNB represented $29.0 \%$ of infections caused by gram negative organisms. Carbapenem resistance, the multi-drug resistant (MDR) phenotype, and MBL production were documented in 70\%, 63\%, and 59\% of NFGNB isolates, respectively. MDR-NFGNB rates were significantly higher among hospitalized patients, medical department and those with longer duration of hospital stay $(p=0.034,0.026,0.019$; respectively) than non MDR-NFGNB. Conclusion: A high level of carbapenem and multi-drug resistance were detected among the non-fermenter pathogens isolated from hospitalized cases and were more frequently encountered in high risk adult cancer patients requiring longer duration of hospitalization. The MDR-NFGNB are constituting important causes of health-care associated infections in cancer patients.

\footnotetext{
${ }^{*}$ Corresponding author.
}

How to cite this paper: El-Mahallawy, H.A., Hamid, R.M.A., Hassan, S.S., Radwan, S. and Saber, M. (2015) The Increased Frequency of Carbapenem Resistant Non Fermenting Gram Negative Pathogens as Causes of Health Care Associated Infections in Adult Cancer Patients. Journal of Cancer Therapy, 6, 881-888. http://dx.doi.org/10.4236/jct.2015.610096 


\section{Keywords}

\section{Multi Drug Resistant Organisms (MDR0), Non-Fermenting Gram Negative Bacilli (NF-GNB), Metallo- $\beta$-Lactamases (MBL), Surgical Site Infections (SSI)}

\section{Introduction}

Non fermenting gram negative bacilli (NFGNB) are taxonomically diverse group of pathogens emerging as a major cause of health-care associated infections (HAI) especially in immunocompromised hosts and patients with malignant disorders [1]. Currently, an increased frequency of serious infections caused by these organisms is reported with a significant contribution to in-hospital mortality [2]. The proper identification of NFGNB up to the species level together with monitoring their susceptibility patterns are mandatory for proper management of infections caused by these pathogens. The antibiotic susceptibility patterns may change with time and may vary from hospital to hospital [3]. Thus, surveillance of the local pattern of HAIs is needed in order to guide appropriate selection of empiric therapy [1].

Carbapenems are considered the last resort for treatment of critically ill patients in ICUs and high risk wards [4]. However, the emergence of increasing frequency of carbapenem-resistant gram negative bacteria, including Pseudomonas aeruginosa and Acinetobacter species, is a threat for medical practice especially in the middleeast region [5]. The resistance to these compounds is mediated by a combination of different mechanisms; with enzymatic hydrolysis, mediated by carbapenemases being the most important mechanism of resistance. Carbapenem hydrolyzing enzymes can be divided into two main sub types; Serine $\beta$-lactamases (Ambler class A and D) and metallo- $\beta$-lactamases (MBLs) (Ambler class B) [6].

Metallo- $\beta$-lactamases are one of the most worrisome resistance mechanisms because in addition to limiting treatment options, their genes are carried on highly mobile elements, allowing easy dissemination [6]. Strains producing MBLs are recorded to be responsible for prolonged HAIs outbreaks associated with serious infections [4]; together with higher morbidity and mortality [7]. Rapid detection of MBLs is essential. This could help to modify therapy and to initiate effective infection control to prevent further dissemination [5]. Thus, we aimed to investigate the prevalence of NFGNB as a cause of health care associated infections in cancer patients and determine their pattern of resistance.

\section{Patients and Methods}

This study was conducted at the Microbiology unit of Clinical Pathology Department of National Cancer Institute (NCI), Cairo University in Egypt, during the period from January 2012 to May 2013. This institute is a tertiary care center receiving an average of 20,000 new cancer cases per year and draining cases from all over the country. The study was approved by the ethical committee review board of NCI, Cairo University with obtaining consents of patients. All the data collected during this study were kept confidential.

\subsection{Data Collection}

The medical records of the patients infected with NFGNB were reviewed for the following data including age, gender, ward of isolation, department (medical or surgical), prior hospitalization, date of admission, duration of hospital stay before onset of infection, clinical site of infection, and clinical outcome within one month of the infectious episode.

\subsection{Microbiology}

A total of 158 non-duplicate NFGNB were isolated out of 544 gram negative organisms cultured from various clinical samples of adult ( $\geq 18$ years) cancer patients. All specimens were collected aseptically and were transported within 2 hours to the laboratory. The isolates were identified according to microscopic examination and cultural characters and identification was done by using Microscan Walk Away 96, Gram-negative panels. The antibiotics panel included amikacin, amoxicillin-clavulanic acid, aztreonam, cefepime, cefotaxime, cefoxitin, ceftazidime, ceftriaxone, ciprofloxacin, gentamicin, imipenem, levofloxacin, meropenem, piperacillin-tazobac- 
tam, tobramycin and trimethoprim-sulfamethoxazole. Results were interpreted according to Clinical and Laboratory Standards Institute (CLSI) guidelines [8], and polymicrobial infections were documented. The isolate was considered as multidrug resistant (MDR), if resistant to more than two classes of antimicrobials ( $\geq 3$ classes) [9].

Imipenem-EDTA Combined disk synergy test (CDST-IPM): Test organisms were inoculated on to plates with Mueller Hinton agar. Two $10 \mu \mathrm{g}$ imipenem discs (Oxoid, UK) were placed on the plate, and appropriate amounts of $10 \mu \mathrm{l}$ of EDTA solution $(0.5 \mathrm{M})$ was added to one of them. The inhibition zones of the imipenem and imipenem-EDTA discs were compared after 24 hours of incubation at $37^{\circ} \mathrm{C}$. The difference of $\geq 7 \mathrm{~mm}$ between the inhibition zone diameter of the imipenem-EDTA disk and that of imipenem disk alone was considered to be positive for the presence of MBLs [4].

Statistical analysis: Data was analyzed using IBM SPSS advanced statistics version 20 (SPSS Inc., Chicago, IL, USA). Numerical data were expressed as mean and standard deviation or median and range as appropriate. Qualitative data were expressed as frequency and percentage. Chi-square test or Fisher's exact test was used to examine the relation between qualitative variables. All tests were two-tailed. A p-value $<0.05$ was considered significant.

\section{Results}

In one and a half year duration, 544 gram negative organism were isolated from microbiology specimens of adult cancer patients; out of which NFGNB were isolated from 158 samples. This accounted for an isolation rate of $29.0 \%$. Acinetobacter baumanii (A. baumannii) was the most common NFGNB isolated (51.9\%), followed by Pseudomonas species (45.6\%), Stenotrophomonas maltophilia (S. maltophilia) (1.3\%), Burkholderia cepacia (B. cepacia) (0.6\%) and Aeromonas hydrophila (A. hydrophila) (0.6\%). The demographic data and clinical characteristics of patients infected with NFGNB are summarized in Table 1. The mean age of patients was $48 \pm 16.5$ years; with $70 \%$ of cases spending $>7$ days prior to infection.

Antimicrobial susceptibility of NFGNB is shown in Figure 1. The results of antimicrobial susceptibility tests of NFGNB showed that $63 \%$ of isolates were MDR $(n=100)$. Table 2 shows the comparison of demographics and clinical characteristics of the cases included in the study between resistant and susceptible groups. Resistant NFGNB were significantly more encountered among female gender, medical department, inpatients, patients with longer duration of hospital stay and $A$. baumannii isolates, with a p value $0.039,0.026,0.034,0.019$, $<0.001$; respectively. Other risk factors including age, prior hospitalization, clinical sites of infections and polymicrobial infections did not significantly differ between both groups, p-value $0.856,0.593,0.573,0.672$; respectively. The MBL phenotype was detected in 59\% of all NFGNB isolates (93/158), by the CDST assay; in 83\% of imipenem resistant isolates (85/102) and $14 \%$ of imipenem sensitive strains (8/56). The MBL positive isolates were significantly more frequently resistant to the other antibiotics (MDR) than MBL-negative isolates (82\% of MDR-NFGNB [82/100] versus 38\% of non MDR-NFGNB [11/58], respectively, with a p-value $<0.001$.

Crude mortality: The crude mortality was recorded in 50 patients among the 158 cases infected by NFGNB (32\%). According to statistical analysis, being infected by MDR-NFGNB was significant predisposing factor for the outcome of patients $(82 \%$ [n = 41], $p=0.004)$.

\section{Discussion}

In the present study, NFGNB constituted 30\% of gram negative pathogens isolated from different clinical specimens of adult cancer patients in one and a half year duration. Similar figures were recorded [7], whilst higher figures (50\%) were also reported [4]. The wide spread use of antibiotics and other chemotherapeutic agents has a major role in the increased frequency of infection by these organisms due to disruption of the normal flora [10]. Pseudomonas and Acinetobacter are the most commonly isolated NFGNB human pathogens [1] [11] [12]. In the present study, NFGNB isolates were A. baumannii, Pseudomonas species, S. maltophilia, B. cepacia and A. hydrophila in $52 \%, 45.6 \%, 1.3 \%, 0.6 \%$, and $0.6 \%$, respectively. In a similar study, Pseudomonas species was the most commonly isolated NFGNB (70\%), followed by Acinetobacter species (30\%) [12]. These differences in the rate of infections may be partly related to the type of host requiring more invasive procedures and partly due to inadequate adherence to infection control policies among different health care settings. Frequent occurrence of hospital infections with $A$. baumannii is often related to antibiotic misuse or overuse and also due to its ability to persist in hospital environment. In case of $A$. baumannii infections, its long life span in hospital premises renders its eradication very difficult [13]. 
Table 1. Demographic data and clinical characteristics of NFGNB infections.

\begin{tabular}{|c|c|c|}
\hline Parameter & Total number & Percentage (\%) \\
\hline \multicolumn{3}{|l|}{ Age, years } \\
\hline $18-40$ & 55 & 34.8 \\
\hline $41-64$ & 70 & 44.3 \\
\hline$\geq 65$ & 33 & 20.9 \\
\hline \multicolumn{3}{|l|}{ Gender } \\
\hline Male & 83 & 52.5 \\
\hline Female & 75 & 47.5 \\
\hline \multicolumn{3}{|l|}{ Department } \\
\hline Surgical & 116 & 73.4 \\
\hline Medical & 42 & 26.6 \\
\hline \multicolumn{3}{|l|}{ ICU admission } \\
\hline No & 118 & 74.7 \\
\hline Yes & 40 & 25.3 \\
\hline \multicolumn{3}{|l|}{ Prior hospitalization } \\
\hline No & 106 & 67.1 \\
\hline Yes & 52 & 32.9 \\
\hline \multicolumn{3}{|l|}{ Hospitalization } \\
\hline Inpatient & 150 & 94.9 \\
\hline Outpatient & 8 & 5.1 \\
\hline \multicolumn{3}{|c|}{ Duration of hospital stay before onset of infection, days } \\
\hline$<7$ & 48 & 30.4 \\
\hline $7-29$ & 78 & 49.4 \\
\hline$\geq 30$ & 32 & 20.2 \\
\hline \multicolumn{3}{|l|}{ clinical site of infection } \\
\hline Blood & 11 & 7.0 \\
\hline Respiratory tract & 61 & 38.6 \\
\hline Urine & 5 & 3.2 \\
\hline Wound swab & 81 & 51.3 \\
\hline \multicolumn{3}{|l|}{ Causative organism* } \\
\hline A. baumannii & 82 & 51.9 \\
\hline Pseudomonas species & 72 & 45.6 \\
\hline S. maltophilia & 2 & 1.3 \\
\hline B. серасіа & 1 & 0.6 \\
\hline A. hydrophila & 1 & 0.6 \\
\hline \multicolumn{3}{|l|}{ Mixed bacterial infections } \\
\hline No & 77 & 48.7 \\
\hline Yes & 81 & 51.3 \\
\hline \multicolumn{3}{|l|}{ Outcome } \\
\hline Alive & 108 & 68.4 \\
\hline Dead & 50 & 31.6 \\
\hline
\end{tabular}

${ }^{*}$ A baumanii = Acinetobacter baumanii, S. maltophilia = Stenotrophomonas maltophilia, B. cepacia = Burkholderia cepacia, A hydrophila = Aeromonas hydrophila . 
Table 2. Comparison of demographics and clinical characteristics between MDR-NFGNB against non MDR-NFGNB.

\begin{tabular}{|c|c|c|c|}
\hline & $\begin{array}{c}\text { Non-MDR }(\mathrm{n}=58) \\
\text { No. }(\%)\end{array}$ & $\begin{array}{l}\text { MDR (n = 100) } \\
\text { No. (\%) }\end{array}$ & p-value \\
\hline Age, years & & & 0.856 \\
\hline $18-40(n=55)$ & $18(33)$ & 37 (67) & \\
\hline $41-64(n=70)$ & $29(41)$ & 41 (59) & \\
\hline$\geq 65(\mathrm{n}=33)$ & $11(33)$ & $22(67)$ & \\
\hline Gender & & & 0.039 \\
\hline Male $(\mathrm{n}=83)$ & $38(46)$ & $45(54)$ & \\
\hline Female $(\mathrm{n}=75)$ & $20(27)$ & 55 (73) & \\
\hline Department & & & 0.026 \\
\hline Surgical (n = 116) & $46(40)$ & $70(60)$ & \\
\hline Medical (n = 42) & $12(29)$ & $30(71)$ & \\
\hline Prior hospitalization & & & 0.593 \\
\hline No $(n=106)$ & $36(34)$ & $70(66)$ & \\
\hline Yes $(n=52)$ & $22(42)$ & $30(58)$ & \\
\hline Hospitalization & & & 0.034 \\
\hline Inpatient $(\mathrm{n}=150)$ & $52(35)$ & $98(65)$ & \\
\hline Outpatient $(\mathrm{n}=8)$ & $6(75)$ & $2(25.0)$ & \\
\hline \multicolumn{2}{|c|}{ Duration of hospital stay before infection, days } & & 0.019 \\
\hline$<7(\mathrm{n}=48)$ & $26(54)$ & $22(46)$ & \\
\hline $7-29(n=78)$ & $25(32)$ & $53(68)$ & \\
\hline$\geq 30(\mathrm{n}=32)$ & $7(22)$ & 25 (78) & \\
\hline Clinical sites of infections & & & 0.573 \\
\hline Blood $(\mathrm{n}=11)$ & $3(27)$ & $8(73)$ & \\
\hline Respiratory tract $(n=61)$ & $19(31)$ & 42 (69) & \\
\hline Urine $(n=5)$ & $2(40)$ & $3(60)$ & \\
\hline Wound swab $(\mathrm{n}=81)$ & $34(42)$ & $47(58)$ & \\
\hline Causative organism & & & $<0.001$ \\
\hline A. baumannii $(\mathrm{n}=82)$ & $8(10)$ & $74(90)$ & \\
\hline Other NFGNB $(n=76)$ & $50(66)$ & $26(34)$ & \\
\hline Mixed bacterial infections & & & 0.672 \\
\hline No $(n=77)$ & $27(35)$ & $50(50)$ & \\
\hline Yes $(\mathrm{n}=81)$ & $31(38)$ & $50(50)$ & \\
\hline Outcome & & & 0.004 \\
\hline Alive $(n=108)$ & $49(45)$ & $59(55)$ & \\
\hline Dead $(n=50)$ & $9(18)$ & $41(82)$ & \\
\hline
\end{tabular}

p values $\leq 0.05$ are considered significant. 


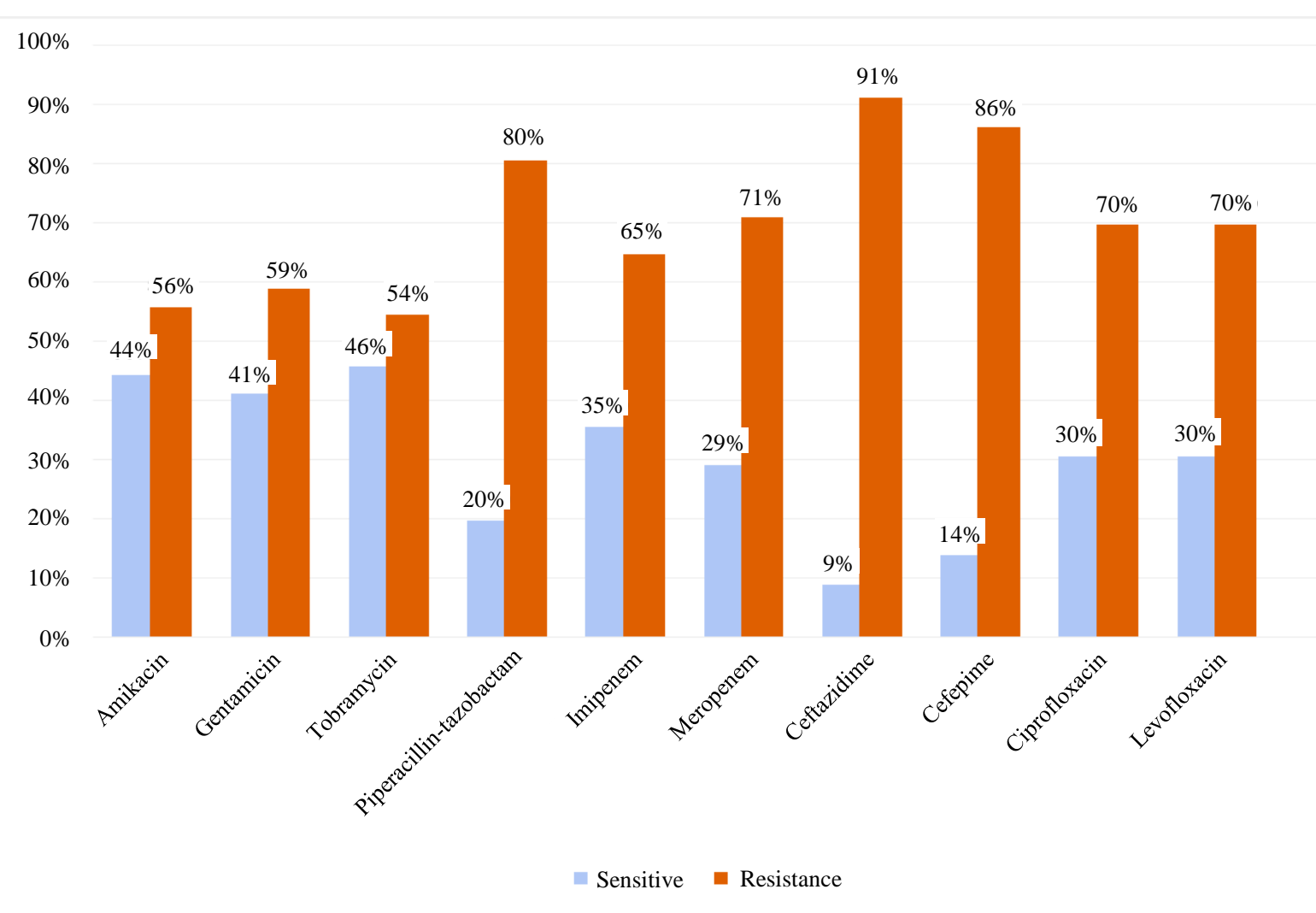

\section{Figure 1. Antibiogram of NFGNB isolates.}

The clinical site of infection is an important epidemiological indicator [9]. Surgical site infections were the most common site of infections in the present study, followed by lower respiratory tract infections. Similar results were previously reported [12]. Of the 158 NFGNB pathogens isolated, polymicrobial growth was detected in 51\% of the specimens. Polymicrobial infections were also reported in 50\% of NFGNB isolates of hospitalized patients [1]. It is evident that cancer patients are more liable to mixed infections. This could be explained by the nature of operations undergone in cancer patients requiring more invasive procedures in contaminated areas, like colorectal, urological and head and neck cancers; besides their immunocompromised status. Also, NCI is a tertiary cancer center draining cases from all over Egypt, so patients might have acquired colonization with NFGNB prior to admission to NCI. Polymicrobial infections have important implications on the clinical course of the disease including limiting the selection of antimicrobial therapy and worsening the response to treatment, especially when it involves pathogens commonly exhibiting antimicrobial resistance [14].

Longer duration of hospital stay and surgical intervention are important risk factors for infection with NFGNB [12]. In our study, 73\% of the patients infected with NFGNB were from surgical wards, 70\% of patients spent more than one week before onset of infection and 95\% of isolated NFGNB were recovered from hospitalized patients. Similarly, 85\% of patients infected with NFGNB were hospitalized [11]. This clarifies the impact of health-care associated infections in acquisition of these types of pathogens. Age seemed to be another risk factor for acquisition of NFGNB infections in the present study, as 65\% of patients infected with NFGNB were above 40 years. It was also documented that $72 \%$ of patients infected with NFGNB were above 45 years [3]. This finding could be due to the fact that cancer is a disease of old age [15].

Carbapenem resistance has become a serious therapeutic problem worldwide [5]. In the current study, high levels of resistance among NFGNB isolates were detected to carbapenems and also to the different antibiotics tested. In general, high rates of MDR are increasingly reported worldwide limiting the options of treatment in high risk patients [1]. High rates of MDR (63\%) were reported in the present study. The evolution of MDR is relatively fast, as the main driving force is lateral gene transfer, a process influenced by a wide range of mobile genetic elements. Integrons have often been found in plasmids and/or transposons that enhance the spread of re- 
sistance genes [16]. In the current study, MDR were significantly higher among females, hospitalized patients, medical department and those with longer duration of hospital stay; $\mathrm{p}=0.039,0.034,0.026$, and 0.019 , respectively. These findings emphasize the role of hospitalization in acquisition of MDR-NFGNB. Similarly, female gender and longer duration of hospital stay were risk factors for acquisition of resistance in NFGNB [17] [18]. Thus, it is evident that resistance to carbapenem and other broad spectrum antibiotics becomes a threat in high risk adult cancer patients whenever longer duration of hospitalization is needed.

The MBL phenotype was detected in 59\% of all NFGNB isolates by the CDST assay in the current study; in $83 \%$ of imipenem resistant isolates and in $14 \%$ of imipenem sensitive strains. Similarly, $73 \%$ of the imipenem resistant NFGNB were MBL producers [5]; however lower rates of MBLs among imipenem resistant NFGNB were also recorded [4]. It is evident that MBLs are increasingly recognized among imipenem resistant isolates, and they also exist in imipenem susceptible isolates. These carbapenem susceptible organisms with hidden MBL genes can spread unnoticed in hospitals if such isolates are reported as sensitive without screening for the presence of MBLs. The treatment of these organisms poses a serious therapeutic challenge as these strains are most often resistant to multiple drugs [19]. The detection of MBLs among imipenem sensitive isolates was reported in some studies with varying percentages [19]. Remarkably, MBL positive isolates were significantly more frequently resistant to the other antibiotics (MDR) than MBL-negative isolates $(\mathrm{p}<0.001)$. A higher prevalence of more resistance was observed in a similar study in MBL positive A. baumannii and $P$. aeruginosa isolates for all antibiotics except colistin. Since MBL genes are carried on plasmids, this may explain the higher prevalence of co-resistance to other antibiotics found in MBL-positive isolates [5].

Thus, non-fermenter gram negative pathogens constituted 30\% of gram negative organisms isolated from clinical infectious specimens of adult cancer patients in one and a half year duration at National Cancer Institute, Cairo University. Non fermenters were primarily isolated from surgical site infections followed by lower respiratory tract infections confirming the role of hospitalization and surgical intervention in transmission of these pathogens. Polymicrobial infections were detected in 50\% of NFGNB isolates of patients. A high level of carbapenem resistance and multi-drug resistance were encountered among the non-fermenter pathogens. The MDR phenotype was significantly more detected in hospitalized patients, medical department and those with longer duration of hospital stay. It could be concluded that antimicrobial resistance to carbapenems and other broad spectrum antibiotics becomes a threat in high risk adult cancer patients requiring longer duration of hospitalization. Also, MDR-NFGNB are constituting important causes of health-care associated infections in cancer patients that are associated with high rates of crude mortality.

Further studies are recommended to characterize the specific factors subjecting cancer patients to be more at risk of these pathogens and also to assign the main lines of preventive measures to control such infections with the changing medical practice towards more invasive procedures.

\section{Acknowledgements}

The authors are thankful to the microbiology staff who undergo the routine microbiology work.

\section{Fund}

The study is done as a part of NCI work and funded by NCI.

\section{Conflict of Interest}

No conflict of interest is considered for any of the authors of this study.

\section{References}

[1] Juyal, D., Prakash, R., Shanakarnarayan, S.A., Sharma, M., Negi, V. and Sharma, N. (2013) Prevalence of Non-Fermenting Gram Negative Bacilli and Their in Vitro Susceptibility Pattern in a Tertiary Care Hospital of Uttarakhand: A Study from Foothills of Himalayas. Saudi Journal for Health Science, 2, 108-112. http://dx.doi.org/10.4103/2278-0521.117915

[2] Karjigi, S., Golia, S. and Reddy, M.K. (2013) Phenotypic Characterization and Antibiotic Susceptibility Pattern of Non-Fermentative Gram Negative Bacilli from Clinical Samples. International Journal of Basic and Applied Medical Sciences, 3, 248-255.

[3] Rit, K., Nag, F., Raj, H.J. and Maity, P.K. (2013) Prevalence and Susceptibility Profiles of Non-Fermentative Gram 
Negative Bacilli Infection in a Tertiary Care Hospital of Eastern India. Indian Journal of Clinical Practice, 24, 451455.

[4] Hodiwala, A., Dhoke, R. and Urhekar, A.D. (2013) Incidence of Metallo-Beta-Lactamase Producing Pseudomonas, Acinetobacter \& Enterobacterial Isolates in Hospitalised Patients. International Journal of Pharmacy and Biological Sciences, 3, 79-83.

[5] Mohanty, S., Maurya, V., Gaind, R. and Deb, M. (2013) Phenotypic Characterization and Colistin Susceptibilities of Carbapenem-Resistant of Pseudomonas aeruginosa and Acinetobacter spp. Journal of Infection in Developing Countries, 7, 880-887. http://dx.doi.org/10.3855/jidc.2924

[6] Omair, M., Usman, J., Kaleem, F., Hassan, A., Khalid, A. and Fahim, Q. (2012) Evaluation of Combined Disc Method for the Detection of Metallo- $\beta$-Lactamase Producing Gram Negative Bacilli. Malaysian Journal of Microbiology, 8, 21-25.

[7] Pandya, N.P., Prajapati, S.B., Mehta, S.J., Kikani, K.M. and Joshi, P.J. (2011) Evaluation of Various Methods for Detection of Metallo- $\beta$-Lactamase (MBL) Production in Gram Negative Bacilli. International Journal of Biological and Medical Research, 2, 775-777.

[8] CLSI (2010) Performance Standards for Antimicrobial Susceptibility Testing; Twentieth Informational Supplement. CLSI Document M100-S20. Clinical and Laboratory Standards Institute, Wayne.

[9] Vaze, N.D., Emery, C.L., Hamilton, R.J., Brooks, A.D. and Joshi, S.G. (2013) Patient Demographics and Characteristics of Infection with Carbapenem-Resistant Acinetobacter baumannii in a Teaching Hospital from the United States. Advances in Infectious Diseases, 3, 10-16. http://dx.doi.org/10.4236/aid.2013.31002

[10] Upgade, A., Prabhu, N., Gopi, V. and Soundararajan, N. (2012) Current Status of Antibiotic Resistant Non-Fermentative Gram Negative Bacilli among Nosocomial Infections. Advances in Applied Science Research, 3, 738-742.

[11] Jayanthi, S. and Jeya, M. (2012) Clinical Distribution and Antibiotic Resistance Pattern of Non-Fermenting Gram Negative Bacilli. International Journal of Pharma and Bio Sciences, 3, 487-494.

[12] Thipperudraswamy, T., Sudhindra, K.S., Nadigar, S. and Kumar, M. (2014) Prevalence of Non-Fermenting Gram Negative Bacilli from Clinical Isolates and Their Antibiogram Profile. Journal of Biomedical and Pharmaceutical Research, 3, 55-61.

[13] Netsvyetayeva, I., Sikora, M., Golas, M., et al. (2011) Acinetobacter baumannii Multidrug-Resistant Strain Occurrence in Liver Recipients with Reference to Other High-Risk Groups. Transplantation Proceedings, 43, 3116-3120. http://dx.doi.org/10.1016/j.transproceed.2011.07.008

[14] Peters, B.M., Jabra-Rizk, M.A., O’May, G.A., Costerton, J.W. and Shirtliff, M.E. (2012) Polymicrobial Interactions: Impact on Pathogenesis and Human Disease. Clinical Microbiology Reviews, 25, 193-213. http://dx.doi.org/10.1128/CMR.00013-11

[15] Ahmad, A.S., Ormiston-Smith, N. and Sasieni, P.D. (2015) Trends in the Lifetime Risk of Developing Cancer in Great Britain: Comparison of Risk for Those Born from 1930 to 1960. British Journal of Cancer, 112, 943-947. http://dx.doi.org/10.1038/bjc.2014.606

[16] Prata-Rocha, M.L., Moreira, M.R., Gontijo Filho, P.P. and Melo, G.B. (2013) Acinetobacter baumannii: Global Evolution of Carbapenem-Resistant and Genotyping Methods. Microbial Pathogens and Strategies for Combating Them: Science, Technology and Education, 1, 197-203.

[17] Rahbar, M., Mehragan, H. and Akbari, N.H.A. (2010) Prevalence of Drug Resistance in Non-Fermenter Gram-Negative Bacilli. Iranian Journal of Pathology, 5, 90-96.

[18] Zahid, M., Akbar, M., Sthanadar, A.A., Ali, P.A., et al. (2014) Isolation and Identification of Multi-Drug Resistant Strains of Non-Lactose Fermenting Bacteria from Clinical Refuses in Major Hospitals of Khyber Pakhtunkhwa, Pakistan. Open Journal of Medical Microbiology, 4, 124-131. http://dx.doi.org/10.4236/ojmm.2014.42014

[19] Daef, E.A., Mohamed, I.S., Ahmed, A.S., Elsherbiny, N.M. and Sayed, I.M. (2012) Evaluation of Different Phenotypic Assays for the Detection of Metallo- $\beta$-Lactamase Production in Carbapenem Susceptible and Resistant Acinetobacter baumannii Isolates. Journal of American Science, 8, 292-299. 